\title{
Biofilm formation in invasive Staphylococcus aureus isolates is associated with the clonal lineage
}

\author{
Preneshni Rochelle Naicker

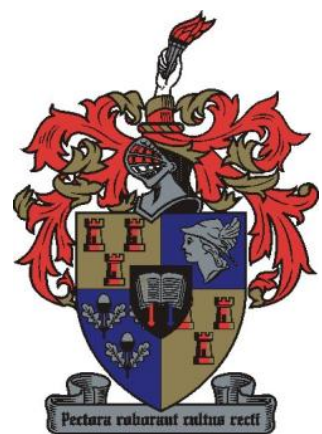 \\ Research article submitted in fulfilment of the requirements for the degree of \\ Master of Medicine (Microbiological Pathology) \\ in the Faculty of Medicine and Health Sciences at Stellenbosch University \\ Supervisor: Professor Elizabeth Wasserman \\ Faculty of Medicine and Health Sciences, Stellenbosch University \\ Co-supervisor: Dr Kim Hoek \\ Faculty of Medicine and Health Sciences, Stellenbosch University
}

February 2014 


\section{Declaration}

I, Preneshni Rochelle Naicker, hereby declare that the work contained in this assignment is my original work and that I have not previously submitted it, in its entirety or in part, at any university for a degree.

Signature:...

Date:. 
Biofilm formation in invasive Staphylococcus aureus isolates is associated with the clonal lineage

S. aureus biofilm formation and genetic background

Preneshni R. Naicker ${ }^{1,2}$, Karayem Karayem ${ }^{1}$, Kim G.P. Hoek ${ }^{1,2}$, Justin Harvey ${ }^{3}$, Elizabeth Wasserman ${ }^{1,4}$

${ }^{1}$ Division of Medical Microbiology, Stellenbosch University, South Africa

${ }^{2}$ National Health Laboratory Services (NHLS) Tygerberg Hospital, Cape Town, South Africa

${ }^{3}$ Centre for Statistical Consultation, Stellenbosch University, South Africa

${ }^{4}$ Pathcare Laboratories and Stellenbosch University, South Africa

Correspondence

Dr Preneshni Naicker

Division of Medical Microbiology

University of Cape Town/NHLS Groote Schuur Hospital

Cape Town, South Africa

preneshni.naicker@nhls.ac.za

\section{Contents Category}

1. Summary (Abstract)

2. Introduction

3. Methods

4. Results

5. Discussion

6. Acknowledgements

7. Conflict of Interest

8. References

9. Addendum 


\begin{abstract}
Objectives: The ability to form biofilms contributes significantly to the virulence of Staphylococcus aureus. Virulence factors may be associated with certain S. aureus lineages. However, the contribution of the genetic background of $S$. aureus to biofilm formation is poorly understood. This study investigated the association between the genetic background and the biofilm forming ability of clinical invasive $S$. aureus isolates. Secondary objectives included investigating any correlation with biofilm formation and methicillin resistance or the source of bacteraemia.
\end{abstract}

Methods: The study was conducted at a 1300-bed tertiary hospital in Cape Town, South Africa. S. aureus isolates obtained from blood cultures between January 2010 and January 2012 were included. Genotypic characterization was performed by PFGE, spa typing, SCCmec typing and MLST. Thirty genotypically unique strains were assessed for phenotypic biofilm formation with the microtitre plate assay. All isolates were tested in triplicate and an average optical density, measured at a wavelength of $490 \mathrm{~nm}$, was determined. The biofilm forming ability of isolates with $\mathrm{A}_{490}>0.17$ was considered 'weak positive' and $\mathrm{A}_{490}>0.34$ 'strong positive'. Isolates with $\mathrm{A}_{490} \leq 0.17$ were considered non-adherent. ANOVA with Bonferroni-adjusted post-hoc tests and $t$-tests were used for statistical analysis of the association between biofilm forming ability and strain characteristics.

Results: Fifty seven percent of isolates were capable of forming biofilms. Weak biofilm formation occurred in $40 \%(\mathrm{n}=12)$ and strong biofilm formation in $17 \%(\mathrm{n}=5)$ of isolates. Thirteen (43\%) of the isolates were classified as non-adherent. All 5 isolates capable of strong biofilm formation belong to one spa clonal complex (spaCC 064). Strains from spa-CC 064 were capable of higher biofilm formation than other spa clonal complexes ( $\mathrm{p}=0.00002)$. These 5 strains belonged to MLST CC5 and CC8. Biofilm formation did not correlate with methicillin resistance and was not related to the source of bacteraemia.

Conclusion: Biofilm formation correlates with the spa clonal lineage in our population of invasive $S$. aureus strains. High biofilm formation was associated with spa-CC 064. MLST CC5 and CC8 strains are capable of strong biofilm formation.

Keywords: Biofilm; Staphylococcus aureus; Genetic background 


\section{INTRODUCTION}

Staphylococcus aureus has ensured its success as an important pathogen worldwide through its versatility, virulence factors and resistance mechanisms. Invasive $S$. aureus infections have a high mortality rate and infections with methicillin-resistant $S$. aureus (MRSA) have even poorer outcomes (Lowy, 1998; Cosgrove \& Fowler, 2008). Biofilm formation is a major virulence factor of $S$. aureus.

A biofilm is 'an assemblage of surface-associated microbial cells that is encased in an extracellular polymeric substance matrix' (Donlan, 2002). These unique communities form on various indwelling medical devices. Bacteria embedded in a biofilm are more resistant to antimicrobials through several mechanisms. Overall antimicrobial penetration is poor, growth-dependent agents have decreased efficacy due to the slower metabolic state of the bacteria and exchange of resistance genes is easier due to the close proximity of cells. Formation of persister-cells, a subpopulation of bacteria that survive antimicrobial treatment, is also a contributing factor (Cramton \& Gotz, 2004).

Due to advances in the medical field, prosthetic devices are increasingly used in patient management. Biofilms may develop on intravascular catheters, prosthetic heart valves and orthopaedic implants. They become reservoirs for persistent infections and foci for metastatic complications such as endocarditis, deep tissue abscesses, septic arthritis, and osteomyelitis (Costerton et al., 1999; Chu et al., 2005). Definitive management of device-related infection frequently requires removal or replacement of the prosthetic material.

The best-described mechanism of biofilm development in $S$. aureus involves the extracellular molecule polysaccharide intercellular adhesin or poly- $N$ acetylglucosamine (PIA/PNAG) (Mack et al., 1996). PIA/PNAG synthesis is regulated by the intercellular adhesion (ica) locus (Cramton et al., 1999). PIA/PNAG and ica-independent biofilm formation, particularly in MRSA, has also been described (Fitzpatrick et al., 2005; O'Neill et al., 2008).However, the relationship between biofilm formation and the genetic background of $S$. aureus is poorly understood. Different clonal lineages of $S$. aureus may have different biofilm forming capabilities. In the recent literature, differences in biofilm formation were found to be due to the staphylococcus protein A (spa) lineage (Atshan et al., 2012a). Other studies found strong biofilm formation correlated with multilocus sequence typing (MLST) clonal complexes (Croes et al., 2009) or Staphylococcal Chromosome Cassette mec (SCCmec) typing (Lim et al., 2013).

This study investigated the association between the genetic background and the biofilm forming ability of clinical invasive $S$. aureus isolates. In addition, we investigated any correlation with biofilm formation and methicillin resistance or source of bacteraemia.

\section{METHODS}

Setting \& Design. This was a prospective, descriptive study conducted at the National Health Laboratory Service (NHLS) Microbiology Laboratory, Tygerberg Hospital, which is a 1300-bed tertiary referral hospital in Cape Town, South Africa. 
Bacterial strains. S. aureus isolates obtained from pure blood cultures between January 2010 and January 2012 were included. Positive blood cultures were identified using the BACTEC 9240 system (Becton Dickinson, USA). Identification of $S$. aureus was done using Mannitol Salt agar (MSA) and DNase agar plates or Vitek 2 (bioMérieux, Marcy 1'Etoile, France). Kirby-Bauer disk diffusion antimicrobial susceptibility testing was performed and Vancomycin Minimum Inhibitory Concentrations (MIC) were determined using ETests (bioMérieux, France) for all MRSA isolates. Clinical and Laboratory Standards Institute (CLSI) 2010 and 2011 interpretative criteria were used. Isolates were stored on Microbeads in cryobroth at $-70^{\circ} \mathrm{C}$ until defrosted and sub-cultured onto blood agar plates for use in this study.

Genotypic characterization. Pulsed-field gel electrophoresis (PFGE) is considered the reference standard for $S$. aureus strain typing and is the most discriminatory typing method (Deurenberg et al., 2007; Oosthuysen et al., 2013). The method described by McDougal et al. was followed (McDougal et al., 2003). The clones were classified based on the number of isolates and unique PFGE types as major (> 10 isolates per PFGE type), intermediate (4-9 isolates per PFGE type) or minor (2-3 isolates per PFGE types). In addition, spa typing and SCCmec typing were also performed (Harmsen et al., 2003; Milheirico et al., 2007). MLST was performed on a representative set of isolates from each major PFGE cluster (Enright et al., 2000).

Isolate selection. From a total collection of 208 non-repeat isolates, blood culture isolates were selected from each major, intermediary and some minor PFGE clusters. Different spa types within the same cluster were also selected. For MRSA isolates, isolates from the same PFGE clusters and spa type were only selected if they were different on SCCmec typing. To include more PFGE clusters, a singleton and different spa types, 2 isolates were included even though MLST data was not available (Table 1).

\begin{tabular}{|c|c|c|c|c|}
\hline MLST CC & MLST ST & spa-CC & spa types & SCCmec \\
\hline $\begin{array}{l}\text { CC1 } \\
(4)\end{array}$ & ST1 (4) & cc174(4) & $\begin{array}{l}\text { t5471, t174, } \\
\text { t8637, t127 }\end{array}$ & - \\
\hline $\begin{array}{l}\text { CC5 } \\
(7)\end{array}$ & $\begin{array}{c}\text { ST5(2) } \\
\text { ST6(2) } \\
\text { ST461 } \\
\text { ST2122(2) }\end{array}$ & $\begin{array}{l}\operatorname{cc002}(5) \\
\operatorname{cc064(2)}\end{array}$ & $\begin{array}{l}\text { t002, t045 (2) } \\
\text { t071, t570, t701, } \\
\text { t2360 }\end{array}$ & I (2) \\
\hline $\begin{array}{l}\text { CC8 } \\
(6)\end{array}$ & $\begin{array}{c}\text { ST8(2) } \\
\text { ST 239(2) } \\
\text { ST612(2) }\end{array}$ & $\begin{array}{l}\operatorname{cc064(4)} \\
\operatorname{cc021(2)}\end{array}$ & $\begin{array}{c}\text { t1443, t1257, } \\
\text { t1476, } \\
\text { t008, t037(2) }\end{array}$ & $\begin{array}{l}\text { III, IV(2), V, } \\
\text { untypeable }\end{array}$ \\
\hline CC12 & ST12 & cc160 & t160 & - \\
\hline $\begin{array}{c}\text { CC15 } \\
(3)\end{array}$ & ST2126(3) & $\operatorname{cc084(3)}$ & $\begin{array}{l}\text { t084, t279, } \\
\text { t094 }\end{array}$ & - \\
\hline $\begin{array}{c}\text { CC22 } \\
\text { (2) }\end{array}$ & ST22(2) & $\operatorname{cc022(2)}$ & t891, t032 & IV \\
\hline
\end{tabular}




\begin{tabular}{|c|c|c|c|c|}
\hline $\begin{array}{c}\text { CC30 } \\
(2)\end{array}$ & ST36, ST1865 & $\operatorname{cc021(2)}$ & t012, t318 & II \\
\hline $\begin{array}{c}\text { CC45 } \\
(2)\end{array}$ & ST45(2) & $\operatorname{cc015/073(2)}$ & t073, t015 & - \\
\hline CC97 & ST97 & cc267 & t359 & - \\
\hline - & - & $\begin{array}{c}\text { cc084 } \\
\text { singleton }\end{array}$ & $\begin{array}{l}\text { t084 } \\
\text { t148 }\end{array}$ & - \\
\hline
\end{tabular}

Table 1. Genotypic characteristics of strains *Number in parentheses (if $>1$ )

Biofilm assay. Thirty genotypically unique strains were assessed for phenotypic biofilm formation with the microtitre plate assay as described by Christensen et al and modified by O'Neill et al (Christensen et al., 1985; O'Neill et al., 2007). Briefly, $S$. aureus was grown overnight in Brain Heart Infusion (BHI) broth. The culture was

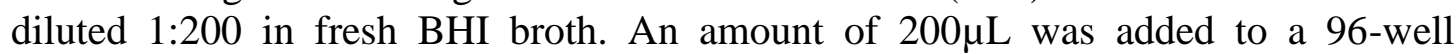
polystyrene tissue-culture treated microtitre plate (Costar® 3595, Corning Incorporated, NY, USA) and incubated at $37^{\circ} \mathrm{C}$ for 24 hours. After overnight incubation the wells were manually rinsed three times with Phosphate-Buffered Saline (PBS), using a hand-held pipette. The plates were air-dried at $60^{\circ} \mathrm{C}$ for one hour to fix the remaining cells. These were then stained with $0.4 \%$ Crystal Violet for two minutes and the plate was rinsed three times with PBS once again. The optical density was measured with a MicroELISA plate reader at a wavelength of 490nm (iMark Microplate Absorbance Reader, Bio-Rad, USA). All isolates were tested in triplicate and an average optical density was determined. A cutoff of 0.17 was used as it was three standard deviations above the mean for a clean tissue culture plate stained as above. Isolates with $\mathrm{A}_{490} \leq 0.17$ were considered non-adherent. $\mathrm{A}_{490}>0.17$ was considered Weak positive and $\mathrm{A}_{490}>0.34$ Strong positive (twice the cutoff value). $S$. epidermidis ATCC 35984 (American Type Culture Collection, Virginia USA) and Brain Heart Infusion broth were used as positive and negative controls respectively.

Clinical data. Clinical information was obtained by clinical consultation with the attending doctor as part of the routine microbiology service for all positive blood cultures. Informed consent was obtained from patients and clinical data was stored in a database with study numbers used for patient confidentiality. Folder reviews were conducted for select cases where insufficient clinical information was gathered. The source of bacteraemia was determined according to the clinical presentation, radiological features and bacteriological factors i.e. the isolation of $S$. aureus from specimens from the site of infection (Orth et al., 2013). The categories were defined as follows: skin and soft tissue (SSTI) was the clinical presentation of soft tissue inflammation together with bacteraemia. Catheter-related blood stream infection (CRBSI) was determined by the culture of $S$. aureus from the catheter tip $\left(>10^{2} \mathrm{cfu}\right)$ and/or clinical evidence of catheter related infection. Pneumonia (PNEUM) was the isolation of S. aureus from the respiratory tract with compatible radiological features. Modified Duke's criteria was used to define infective endocarditis (IE). Bone and joint (B\&J) was clinical and radiological features of osteomyelitis or septic arthritis. Vascular (VASC) was clinical evidence of endovascular infection and intraabdominal (IA) was defined as clinical and radiological evidence of deep-seated intraabdominal source with $S$. aureus cultured from the site (Liao et al., 2008). 
Statistical analysis. ANOVA with Bonferroni-adjusted post-hoc tests and $t$-tests were used for statistical analysis (Statistica version 10, 2011). The biofilm forming ability was analyzed by methicillin resistance, source of bacteraemia, spa, SCCmec and MLST type. Statistical significance was defined as $\mathrm{p}<0.05$.

Ethics. This study received ethical approval from the Division of Research Development and Support, Stellenbosch University (N09/01/012).

\section{RESULTS}

\section{A. Description of isolates}

The 30 isolates selected comprised of 19 PFGE clusters, with 28 spa types and 15 MLST sequence types (ST). These were from 9 spa-clonal complexes (spa-CC) and 9 MLST clonal complexes (MLST CC) (Table 2). 


\begin{tabular}{|c|c|c|c|c|c|c|c|c|}
\hline $\begin{array}{c}\text { MLST } \\
\text { CC }\end{array}$ & $\begin{array}{l}\text { MLST } \\
\text { ST }\end{array}$ & spa-CC & $\begin{array}{l}\text { spa } \\
\text { type }\end{array}$ & $\begin{array}{l}\text { PFGE } \\
\text { cluster }\end{array}$ & $\begin{array}{c}\text { SCCmec } \\
\text { (for MRSA) }\end{array}$ & Clinical* $^{*}$ & $\begin{array}{l}\text { Average } \\
\text { OD }\end{array}$ & Biofilm \\
\hline 1 & 1 & cc174 & $\mathrm{t} 127$ & Z & & SSTI & 0.19 & WEAK \\
\hline 1 & 1 & cc174 & $\mathrm{t} 5471$ & Z & & CRBSI & 0.22 & WEAK \\
\hline 1 & 1 & cc174 & $\mathrm{t} 174$ & AF & & SSTI & 0.14 & NON \\
\hline 1 & 1 & cc174 & t8637 & AF & & B\&J & 0.19 & WEAK \\
\hline 5 & 5 & cc002 & t045 & $M$ & I & CRBSI & 0.15 & NON \\
\hline 5 & 5 & cc002 & $\mathrm{t} 570$ & $\mathrm{Y}$ & & B\&J & 0.12 & $\mathrm{NON}$ \\
\hline 5 & 6 & cc064 & t2360 & $\mathrm{AE}$ & & CRBSI & 1.06 & STRONG \\
\hline 5 & 6 & cc064 & t701 & $\mathrm{AE}$ & & CRBSI & 0.39 & STRONG \\
\hline 5 & 461 & cc002 & t045 & $\mathrm{N}$ & I & CRBSI & 0.1 & NON \\
\hline 5 & 2122 & cc002 & t002 & W & & SSTI & 0.2 & WEAK \\
\hline 5 & 2122 & cc002 & t071 & W & & VASC & 0.18 & WEAK \\
\hline 8 & 8 & cc064 & $\mathrm{t} 1476$ & $Q$ & & VASC & 0.51 & STRONG \\
\hline 8 & 8 & cc064 & t008 & $\mathrm{O}$ & V & SSTI & 0.13 & NON \\
\hline 8 & 239 & cc021 & t037 & $U$ & III & SSTI & 0.18 & WEAK \\
\hline 8 & 239 & cc021 & t037 & $U$ & untypeable & PNEUM & 0.12 & NON \\
\hline 8 & 612 & cc064 & $\mathrm{t} 1443$ & $S$ & IV & CRBSI & 0.39 & STRONG \\
\hline 8 & 612 & cc064 & $\mathrm{t} 1257$ & $S$ & IV & SSTI & 0.51 & STRONG \\
\hline 12 & 12 & cc160 & $\mathrm{t} 160$ & $A B$ & & PNEUM & 0.2 & WEAK \\
\hline 15 & 2126 & cc084 & t084 & AA & & CRBSI & 0.12 & NON \\
\hline 15 & 2126 & cc084 & t279 & $\mathrm{AA}$ & & IE & 0.25 & WEAK \\
\hline 15 & 2126 & $\operatorname{cc084}$ & t094 & AA & & SSTI & 0.12 & NON \\
\hline 22 & 22 & cc022 & t891 & G & & SSTI & 0.18 & WEAK \\
\hline 22 & 22 & cc022 & t032 & G & IV & IA & 0.26 & WEAK \\
\hline 30 & 36 & cc021 & t012 & B & II & SSTI & 0.19 & WEAK \\
\hline 30 & 1865 & $\operatorname{cc021}$ & t318 & $A$ & & IE & 0.15 & NON \\
\hline 45 & 45 & cc015/073 & t073 & $\mathrm{D}$ & & IA & 0.12 & NON \\
\hline 45 & 45 & cc015/073 & t015 & $\mathrm{D}$ & & SSTI & 0.09 & NON \\
\hline
\end{tabular}


Stellenbosch University http://scholar.sun.ac.za

\begin{tabular}{|c|c|c|c|c|c|c|c|c|}
\hline 97 & 97 & $\mathrm{cc} 267$ & $\mathrm{t} 359$ & $\mathrm{P}$ & & CRBSI & 0.13 & NON \\
\hline & & $\mathrm{cc084}^{\#}$ & $\mathrm{t} 084$ & SINGL $^{\#}$ & & CRBSI & 0.3 & WEAK \\
\hline & & SINGL $^{\#}$ & $\mathrm{t} 148$ & $\mathrm{I}$ & & PNEUM & 0.1 & NON \\
\hline
\end{tabular}

Table 2: Genotypic characterization and biofilm formation

* $(\mathrm{SSTI}=$ skin and soft tissue CRBSI $=$ catheter-related blood-stream infections; $\mathrm{PNEUM}=$ pneumonia; $\mathrm{B} \& \mathrm{~J}=$ bone and joint;

IA = intra-abdominal; VASC = vascular; IE = infective endocarditis)

\#Singleton 
There were 9 MRSA (30\%) and 21 methicillin-sensitive S. aureus (MSSA) (70\%). The source of infection was determined for all the $S$. aureus bacteraemias (Figure 1). Ten originated from skin and soft tissue infections (SSTI), 9 from catheter-related blood-stream infections (CRBSI), 3 pneumonias (PNEUM), and 2 each from infective endocarditis (IE), bone and joint (B\&J), vascular (VASC) and intra-abdominal (IA).

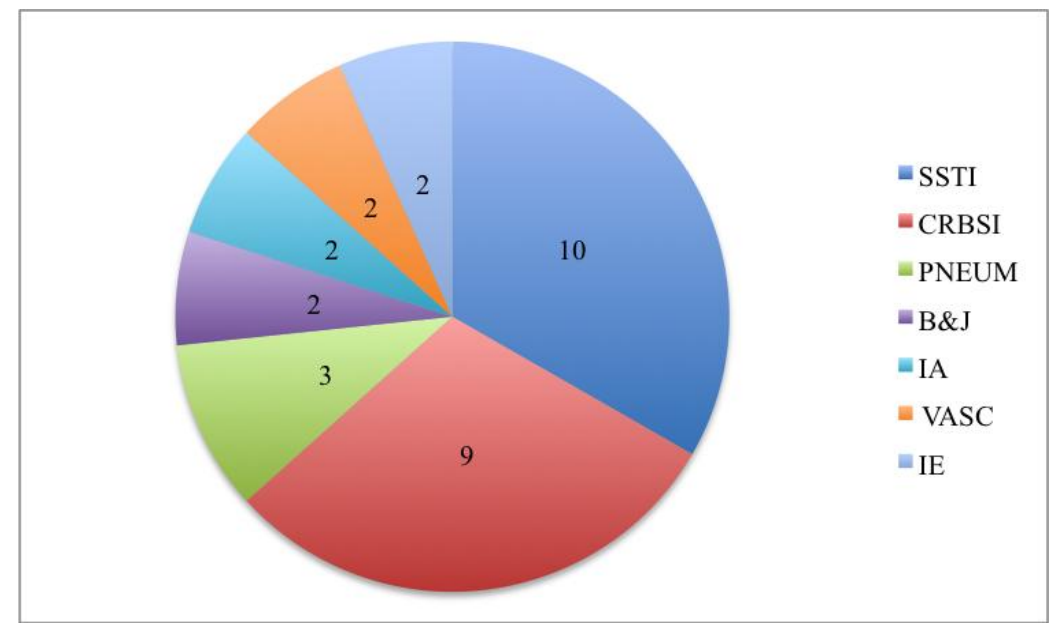

Figure 1. Source of bacteraemia

$(\mathrm{SSTI}=$ skin and soft tissue; $\mathrm{CRBSI}=$ catheter-related blood-stream infections; PNEUM = pneumonia; $\mathrm{B} \& \mathrm{~J}=$ bone and joint; IA = intra-abdominal; VASC = vascular; IE = infective endocarditis)

Biofilm formation occurred in $57 \%$ of isolates (Figure 2). Weak biofilm formation occurred in $40 \%(n=12)$ and strong biofilm formation in $17 \%(n=5)$ of isolates. Thirteen isolates were classified as non-adherent. The reproducibility of the assay was excellent.

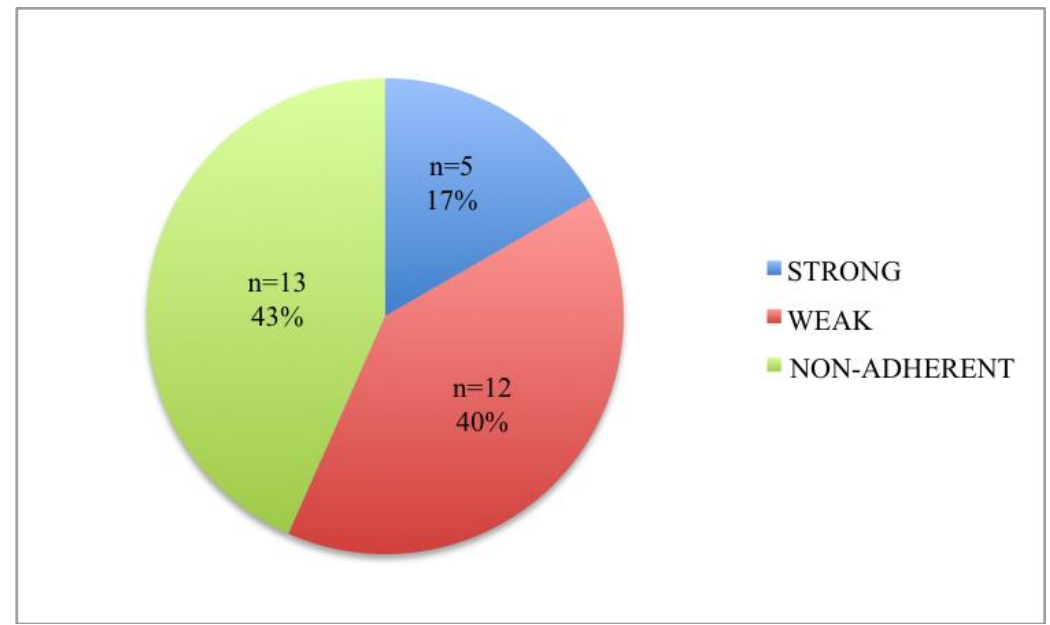

Figure 2. Biofilm categories

\section{B. Analysis by genetic background.}

\section{i) spa typing}

The spa types $\mathrm{t} 701, \mathrm{t} 1275, \mathrm{t} 1443, \mathrm{t} 1476$ and $\mathrm{t} 2360$ were strong biofilm producers. There were too many individual spa types for further analysis. 
All 5 isolates capable of strong biofilm formation belong to one spa clonal complex (spa-CC 064). Strains from spa-CC 064 were capable of higher biofilm formation than all other spa clonal complexes $(\mathrm{p}=0.00002)$ (Figure 3).

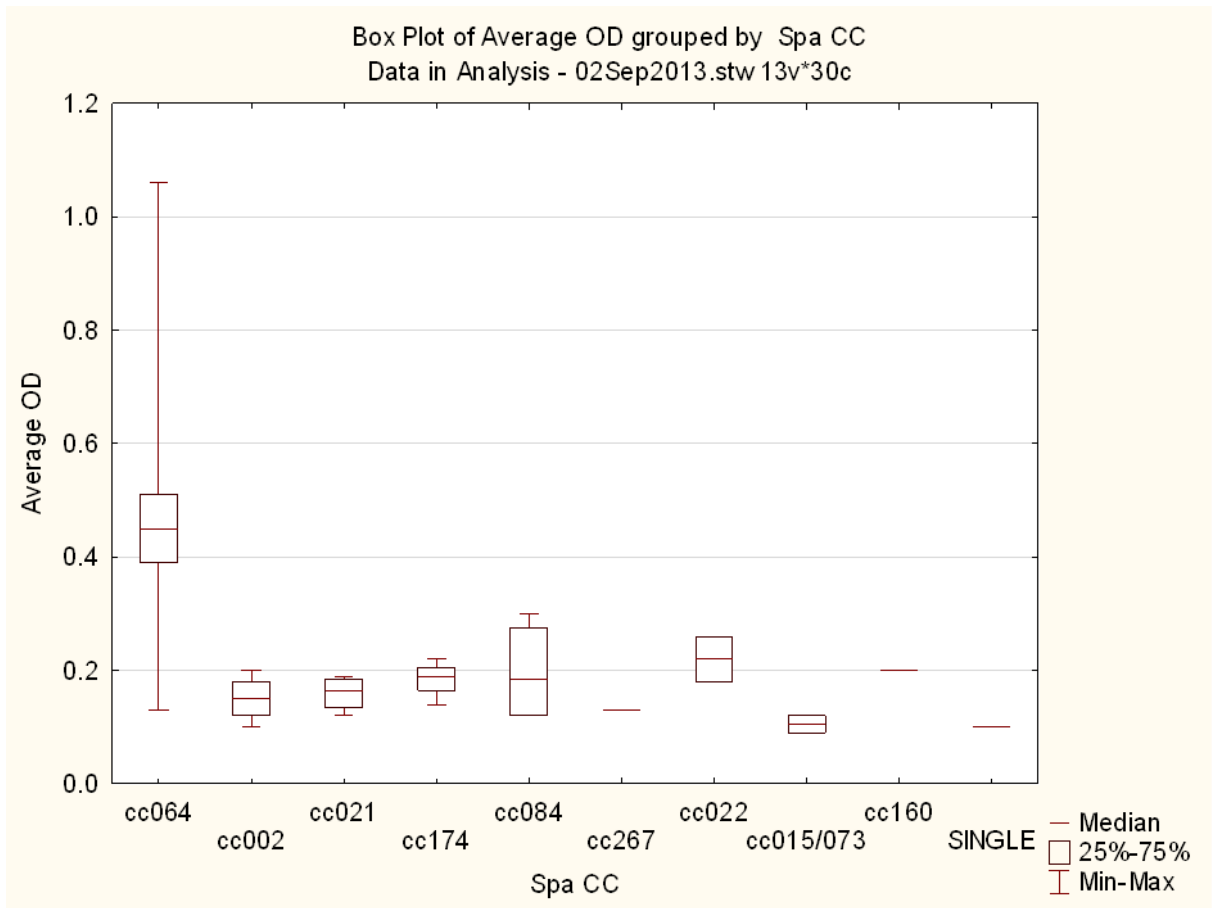

Figure 3. Optical density and spa-CC

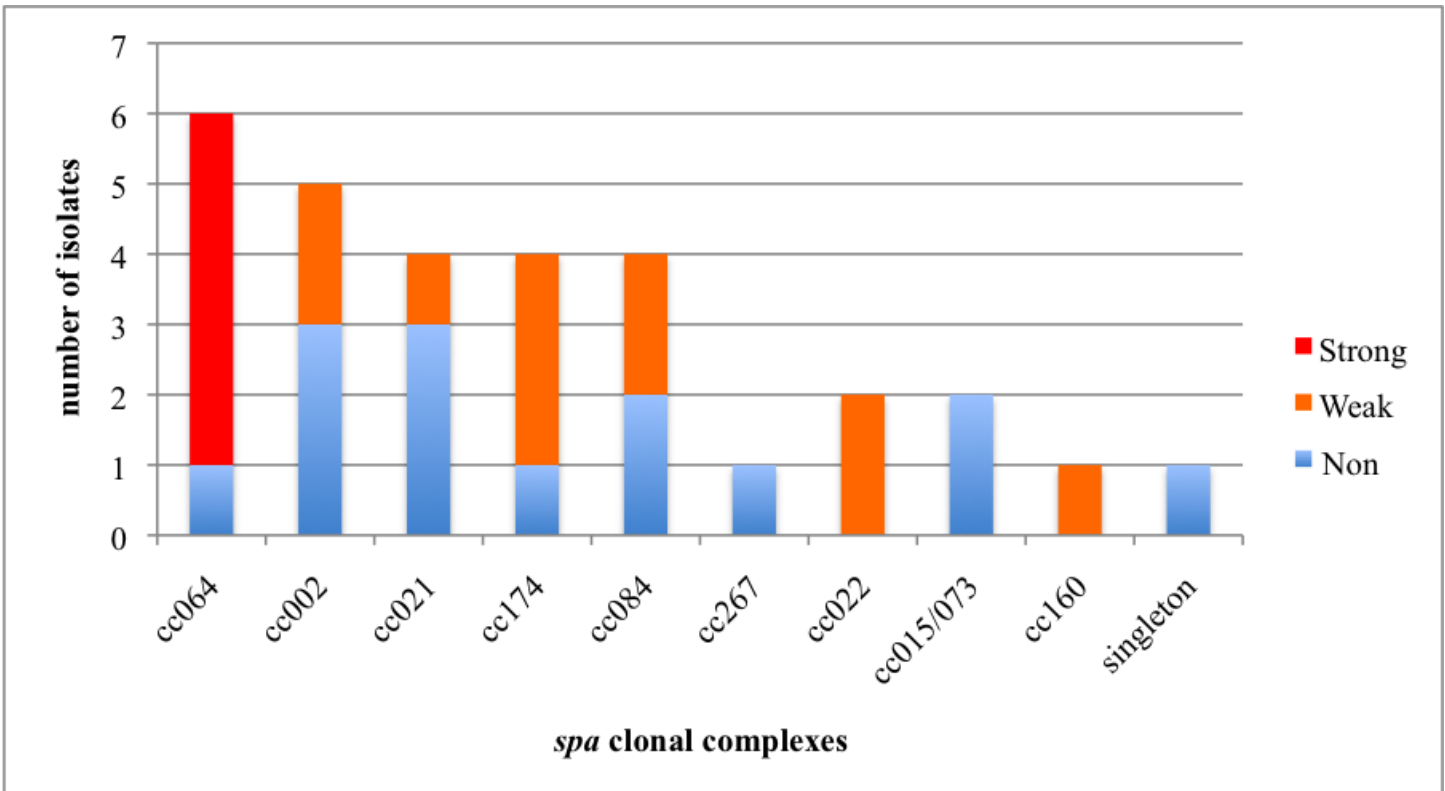

Figure 4. Biofilm categories and spa-CC

\section{ii) MLST}

MLST ST6, ST8 and ST612 produced strong biofilms. Generally the category of biofilm production correlated with the MLST sequence types. 
Strains from MLST CC 1, 5, 8, 12, 15, 22 and 30 were capable of biofilm formation. Although there was no difference found with biofilm formation and MLST CC ( $\mathrm{p}=0.89$ ), MLST CC5 and CC8 isolates had a higher biofilm forming capacity. The 5 strong biofilm producers (all from spa-CC064) fell into MLST CC5 and CC8 (Figure $5 \& 6)$.

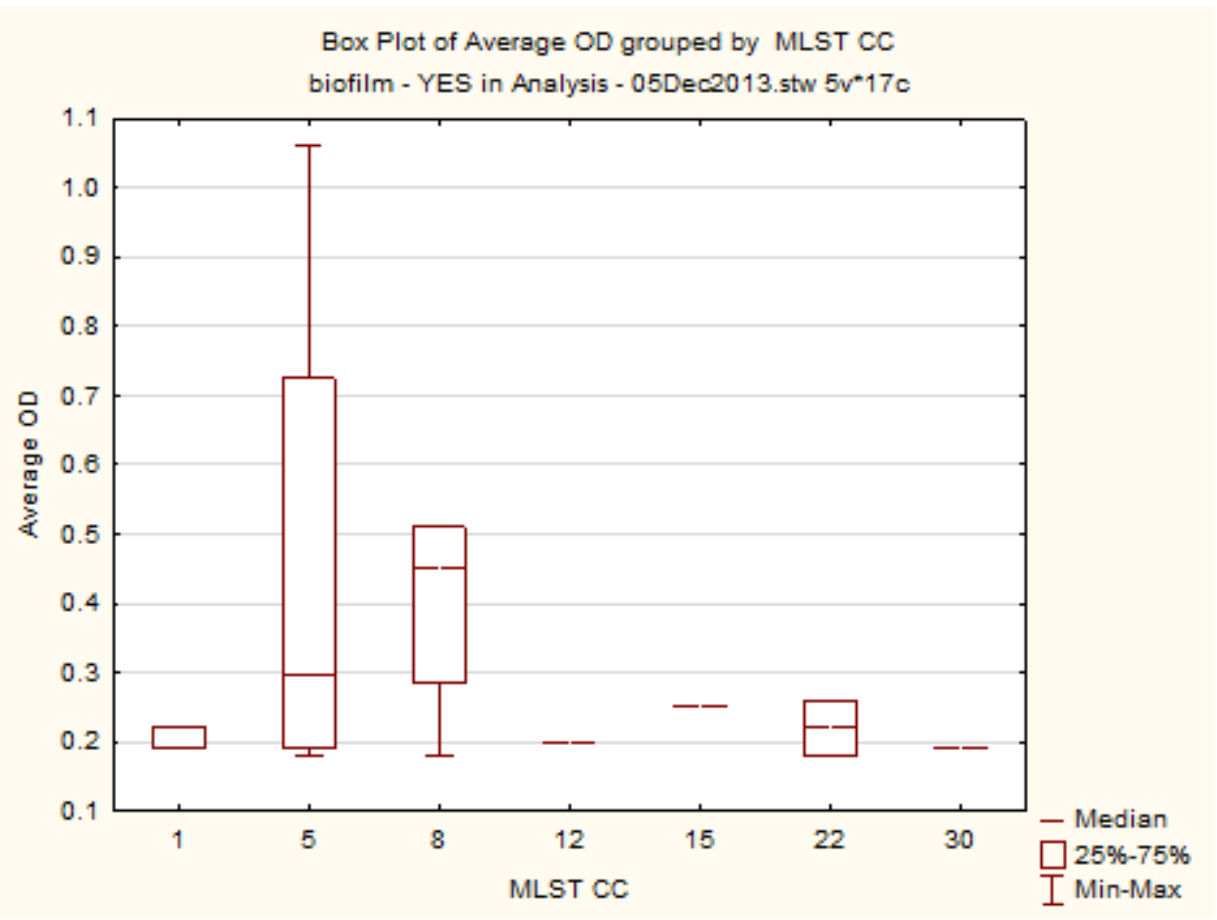

Figure 5. Average OD and MLST CC

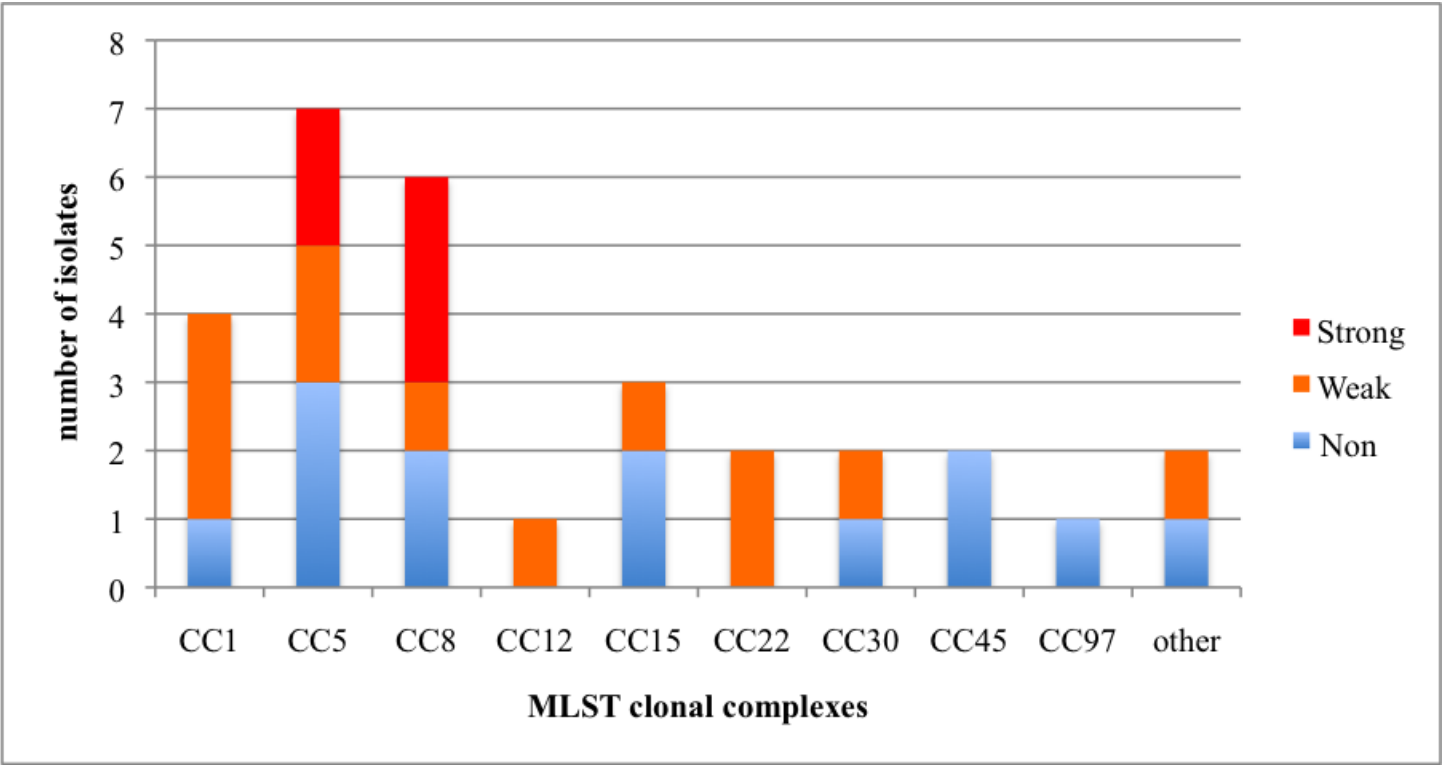

Figure 6. Biofilm categories by MLST CC 


\section{iii) SCCmec typing}

There were only 9 MRSA isolates for SCCmec typing and further analysis could not be performed.

\section{iv) PFGE}

As PFGE strain typing was the basis of selection of isolates, biofilm formation could not be analysed by PFGE clusters due to the large variation.

\section{Analysis by methicillin resistance}

There were no significant differences observed in biofilm formation of MRSA isolates compared with MSSA isolates $(\mathrm{p}=0.9)$ (Figure $7 \& 8$ ).

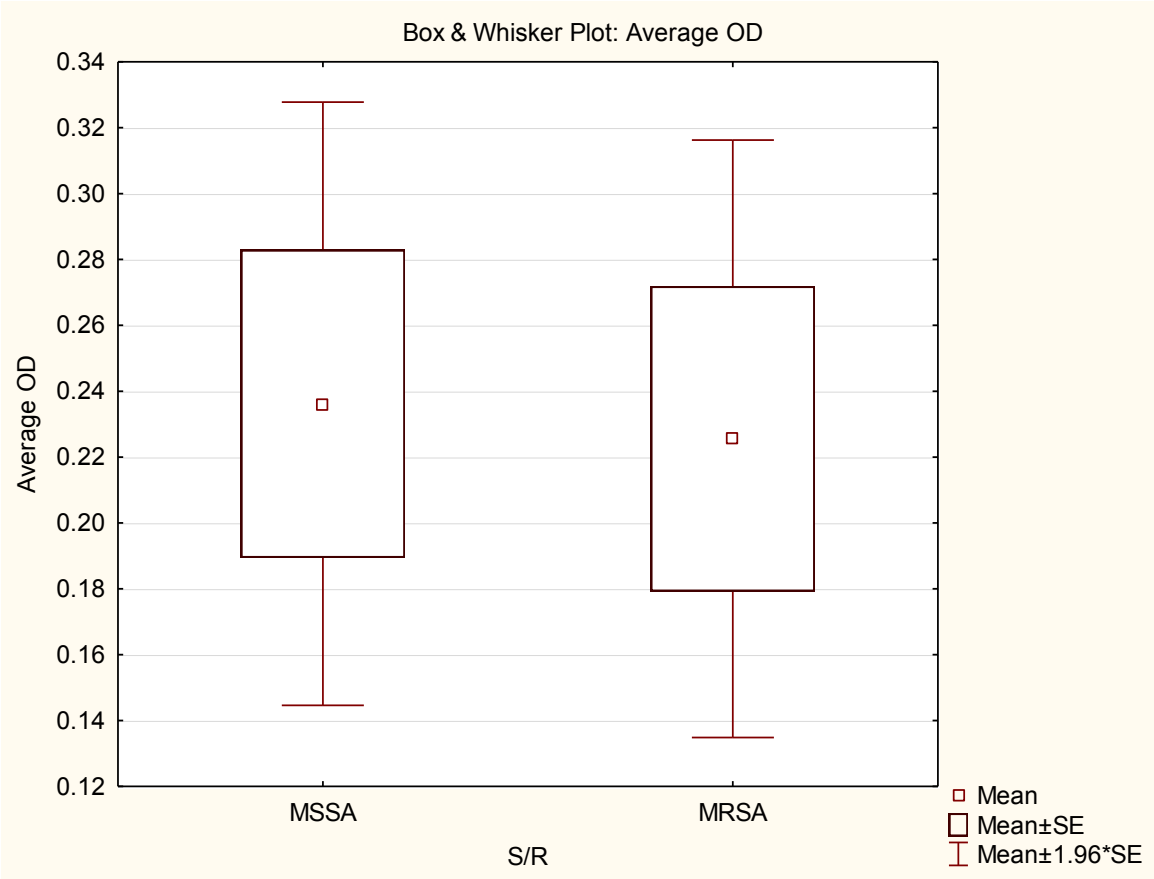

Figure 7. Optical density for MRSA and MSSA isolates 


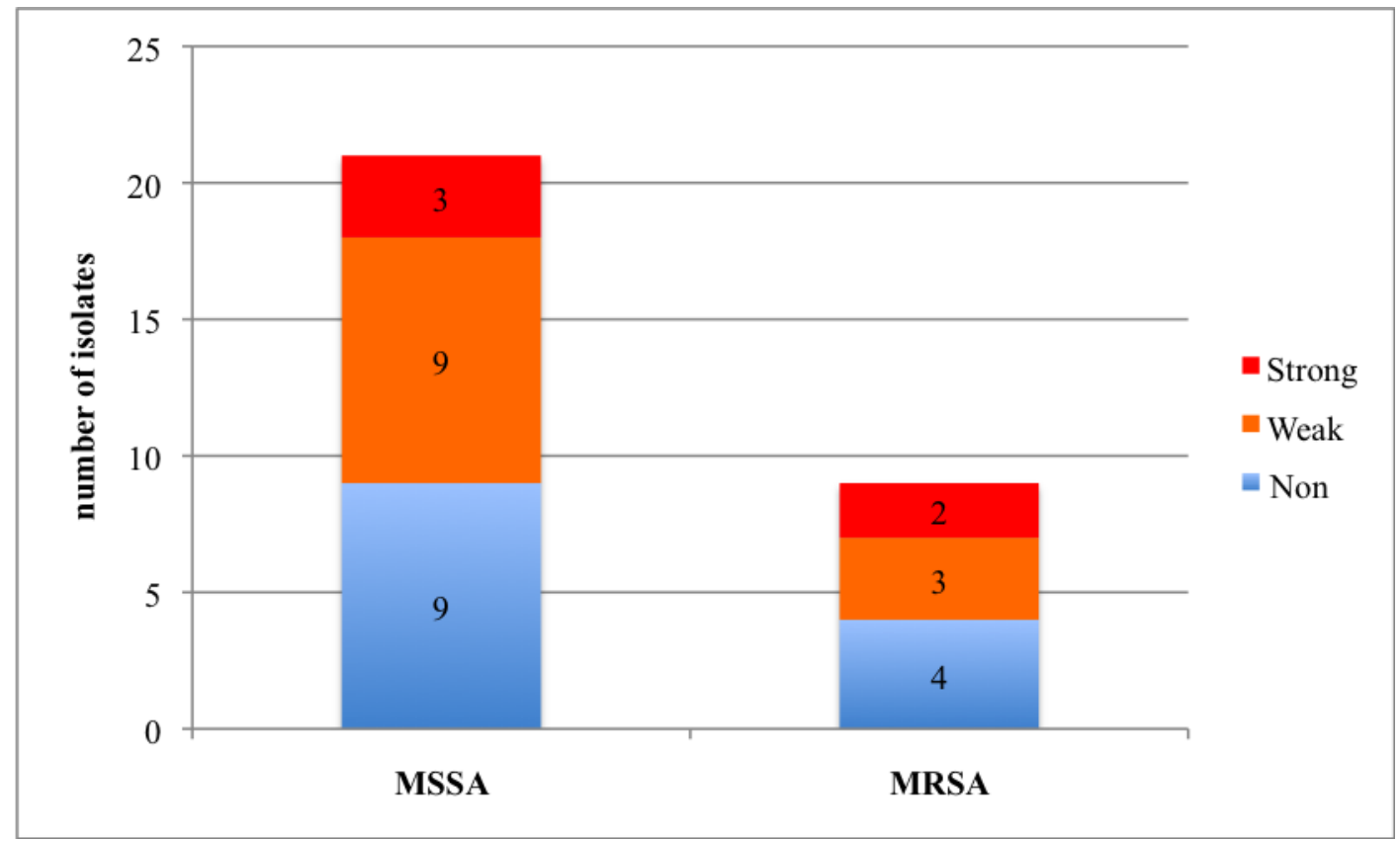

Figure 8. Biofilm categories by Methicillin resistance

\section{Analysis by source of bacteraemia}

There were no differences in biofilm formation relating to the source of bacteraemia $(\mathrm{p}=0.64)$. However, as clinically expected, it was observed that VASC and CRBSI infections had higher median optical densities. The sources of bacteraemia for strong biofilm producers were CRBSI, SSTI and VASC (Figure $9 \& 10$ ).

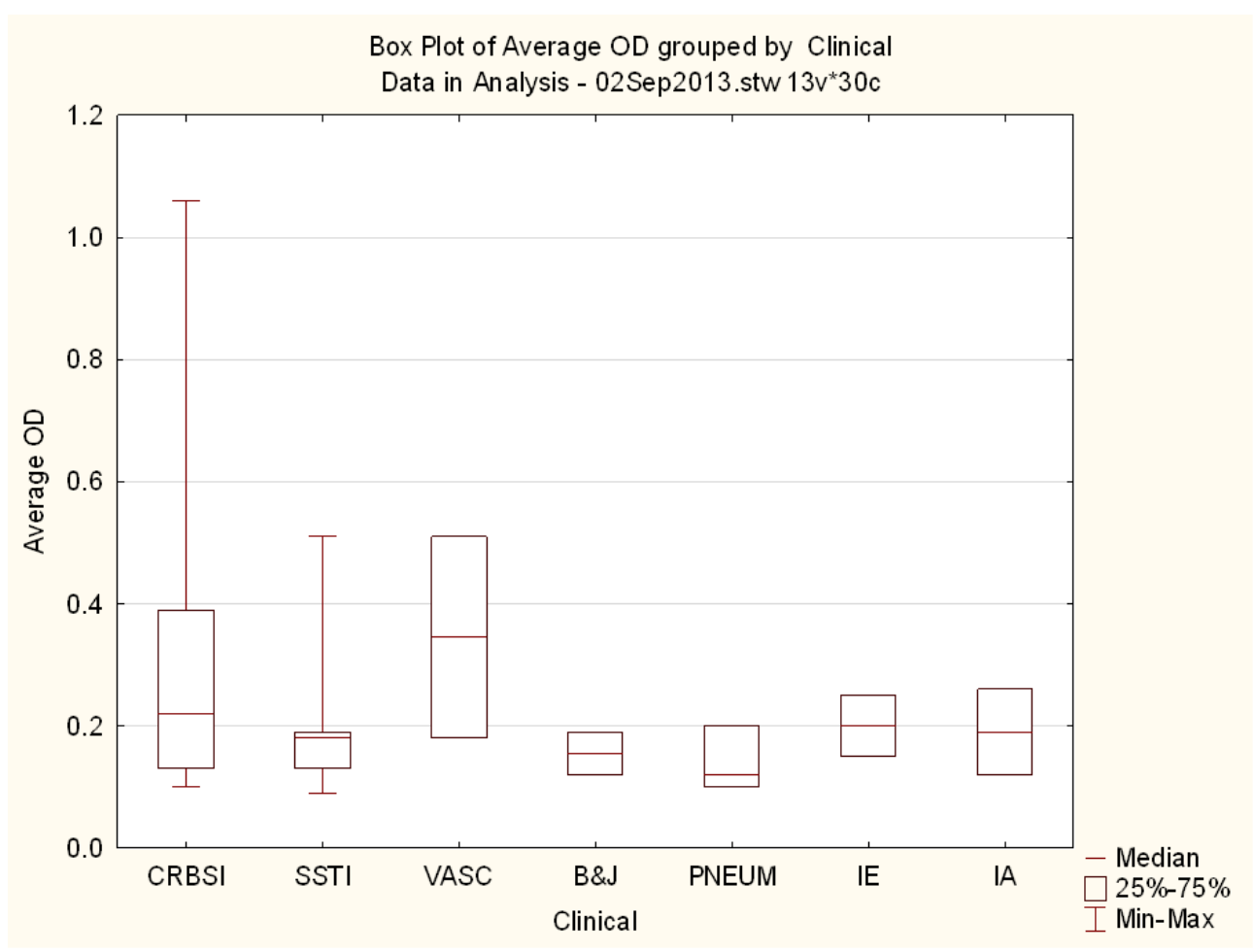

Figure 9. Optical density and source of bacteraemia 


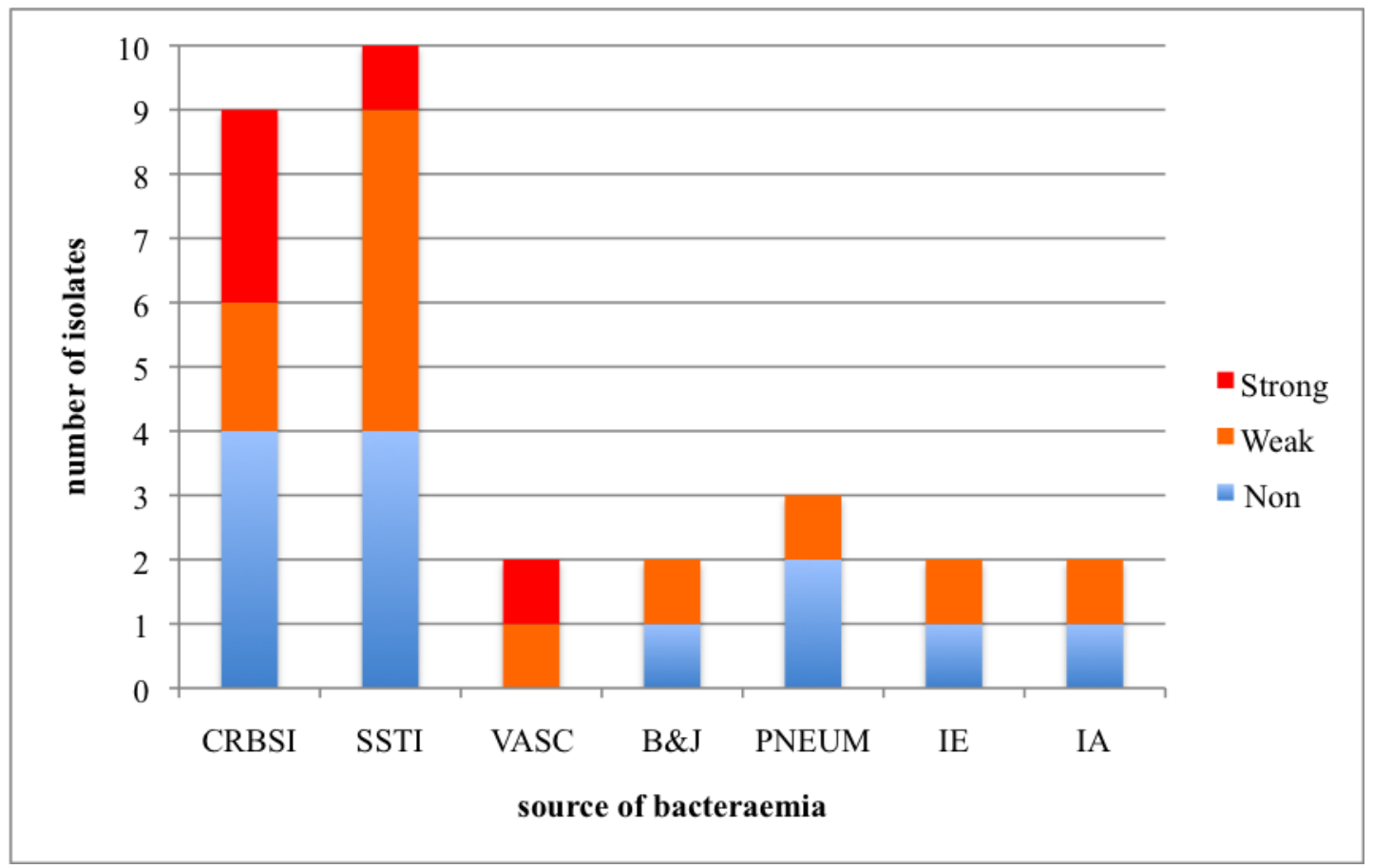

Figure 10. Biofilm categories and source of bacteraemia

\section{DISCUSSION}

Biofilm-associated infections caused by $S$. aureus are a significant cause of morbidity and mortality (Archer et al., 2011). In this study strong biofilm formation was associated with strains from spa-CC 064 and MLST CC 5 and 8. Although MLST CC8 has now been incorporated into MLST CC5, it is considered separately in this analysis (Oosthuysen et al., 2013). As MLST CC8 in now incorporated into MLST CC5, MLST CC5 may be associated with high biofilm formation. This suggests that certain clones are more prone to biofilm formation and that spa-typing and MLST may be a predictor of biofilm formation.

S. aureus demonstrates predominantly clonal evolution. Virulence factors may be associated with specific $S$. aureus lineages. Certain virulence factors such as collagen adhesion (CNA) and toxic shock syndrome toxin 1 (TSST1) are known to be associated with certain clonal lineages (Deurenberg et al., 2009). Therefore some clones are considered more virulent than others (Melles et al., 2004). Biofilm formation is also a major virulence factor and several other observations have supported the link between the clonal lineage and biofilm formation.

Atshan et al. found that strains from the same spa type had similar adherence properties on the microtitre plate assay. In another study by the same group, MRSA strains from the same MLST, spa and SCCmec type had similar biofilm forming abilities, however there was more variation by spa type. In contrast to our findings, they found spa type t037 (ST239-CC8-IIIA) strains form strong biofilms (Atshan et al., 2012a,b). Croes et al. suggested that MLST CC8 was a predisposing factor for a strain to produce strong biofilms (Croes et al., 2009). Furthermore, the Brazilian clone (also MLST CC8) was shown to have increased adherence (Amaral et al., 2005). The 
EMRSA-15 clone in Scotland was found to form stronger biofilms than EMRSA-16 isolates (Smith et al., 2008).

In two South African academic hospitals it was shown that MRSA bacteraemia had a higher mortality rate than MSSA (Perovic et al., 2006). The prevalence of MRSA in bacteraemia at Tygerberg hospital has been reported to be 30\% (Orth et al., 2013), which is reflected in the percentage of MRSA in the collection of strains that we tested. Certain pandemic MRSA clones are spread worldwide. Recent publications describe the ST612-MRSA-IV clone (MLST CC8) as the most widespread MRSA clone in Cape Town, and South Africa (Jansen van Rensburg et al., 2011; Orth et al., 2013; Oosthuysen et al., 2013; Moodley et al., 2010). In this study ST 612 formed strong biofilms, which may contribute to its dominance in nosocomial infections in South Africa.

Some studies found SCCmec typing a predictor of biofilm formation. SCCmec IV isolates were capable of higher biofilm formation (Kwon et al., 2008). In contrast to the findings by Lim et al. where SCCmec III was found to be a genetic risk factor for strong biofilm formation (Lim et al., 2013), we found SCCmec IV isolates produced the strongest biofilms (only three isolates tested). In our study the one SCCmec III isolate did form a weak biofilm. The differences found could also be attributed to the biofilm assay methodology. A larger collection of MRSA isolates should be tested to make any meaningful conclusions.

Kwon et al. demonstrated that MRSA clinical isolates had a greater likelihood of developing biofilms. Approximately $37.9 \%$ of the 66 MRSA formed biofilms compared to $14.3 \%$ of the 35 MSSA tested ( $\mathrm{p}<0.05$ ) (Kwon et al., 2008). Other studies showed no difference in biofilm formation between MRSA and MSSA isolates (Smith et al., 2008; Indrawattana et al., 2013). In our study there was also no difference in biofilm formation between MRSA and MSSA isolates, although the mean optical density for MSSA was higher. Atshan et al. also found MSSA clones had slightly higher biofilm formation than MRSA. Croes et al. found that MRSA or MSSA with an MRSA associated CC, were more capable of strong biofilm formation in the presence of $0.1 \%$ glucose. Our study did not supplement glucose which may have affected the MRSA biofilm capabilities. Our selection criteria was based on the genotypic classification which is also a limiting factor as some clones may be associated with methicillin-resistance (Deurenberg et al., 2007).

Several studies have investigated the source of isolates as a predictor of biofilm formation. Isolates from blood cultures showed a higher frequency of biofilm formation compared to isolates from other sites (Kwon et al., 2008). In the study by Smith et al., the isolates derived from skin had a greater ability to form fully established biofilms (Smith et al., 2008). In our study, the source of bacteraemia as established by clinical data for $S$. aureus blood-stream infections was not a predictor of biofilm formation. However, we only tested blood culture isolates, these being the most clinically important specimens, and did not collect isolates from the source of infection.

The variations in biofilm capacity may be due to differences in surface proteins or gene expression in different $S$. aureus clonal lineages. Microarray analysis showed variation between different lineages is, in particular, due to surface adhesion genes 
and their regulators. However there were no specific genes to differentiate carriage versus invasive isolates (Lindsay et al., 2006). Kuhn et al. also found no difference with adhesion genes in epidemic and sporadic MRSA clones (Kuhn et al., 2006). Gene expression studies and whole genome sequencing may assist to elucidate the reason for these differences (Costa et al., 2013).

The strength of this study was that biofilm formation was tested on a collection of genotypically well characterized strains, all of them isolated from blood cultures. They were selected to be genetically diverse. PFGE, the gold standard for $S$. aureus strain typing, was the basis of the selection to ensure that we did not test the same strain as outbreaks of MRSA may occur in hospital settings. Strains within the same PFGE cluster were chosen if they were different spa or SCCmec types. The limitations were the small numbers included. By testing more isolates we could have also assessed if certain spa-types are more prone to biofilm formation. Another limiting factor was not using supplementary glucose in the biofilm assay. This may influence the MRSA biofilm formation (Croes et al., 2009; O'Neill et al., 2007). Although we did analyse our collection by methicillin resistance and source of bacteraemia, these were secondary objectives. The limitation of selecting isolates based on their genetic background is that conclusions cannot be made about methicillin resistance and source of bacteraemia. Further studies should be conducted with different selection criteria to specifically address these questions. Further investigations for the presence of adhesion genes, gene expression and accessory gene regulator $(a g r)$ groups are required.

Biofilm formation correlates with genetic background in our population of invasive $S$. aureus strains. The propensity to form biofilms may be linked to the epidemic potential of certain successful clones of $S$. aureus. The spa lineage may serve as a genetic predictor of biofilm formation. MLST can also be used to determine clones with higher biofilm formation. Further studies are required to identify these clones with high biofilm formation and investigate the prevalence and expression of adhesion genes.

\section{ACKNOWLEDGEMENTS}

This study was funded by the National Health Laboratory Service (NHLS) Research Trust. We are also grateful to the staff at NHLS Microbiology Tygerberg Hospital and to Mediclinic for funding laboratory equipment.

\section{CONFLICT OF INTEREST}

The authors have no conflicts of interest to declare. 


\section{REFERENCES}

Amaral MM, Coelho LR, Flores RP, Souza RR, Silva-Carvalho MC, Teixeira LA, Ferreira-Carvalho BT, Figueiredo AMS. (2005). The predominant variant of the Brazilian Epidemic Clonal Complex of methicillin-resistant Staphylococcus aureus has an enhanced ability to produce biofilm and to adhere to and invade airway epithelial cells. J Infect Dis 192, 801-10.

Archer NK, Mazaitis MJ, Costerton JW, Leid JG, Powers ME, Shirtliff ME. (2011). Staphylococcus aureus biofilms: Properties, regulation and roles in human disease. Virulence 2, 445-59.

Atshan SS, Shamsudin MN, Lung LTT, Sekawi Z, Ghaznavi-Rad E, Pei CP. (2012a). Comparative characterisation of genotypically different clones of MRSA in the production of biofilms. J Biomed Biotechnol 417247 doi: 10.1155/2012/417247

Atshan SS, Shamsudin MN, Sekawi Z, Lung LTT, Hamat RA, Karunanidhi A, Ali AM, Ghaznavi-Rad E, Ghasemzadeh-Moghaddam H, Seng JSC \& other authors (2012b). Prevalence of adhesion and regulation of biofilm-related genes in different clones of Staphylococcus aureus. J Biomed Biotechnol 976972 doi: $10.1155 / 2012 / 976972$

Christensen GD, Simpson WA, Younger JJ, Baddour LM, Barrett FF, Melton DM, Beachey EH. (1985). Adherence of coagulase-negative Staphylococci to plastic tissue culture plates: a quantitative model for adherence to Staphylococci to medical devices. J Clin Microbiol 22, 996-1006.

Chu VH, Crosslin DR, Friedman JY, Reed SD, Cabell CH, Griffiths RI, Masselink LE, Kaye KS, Corey GR, Reller LB. \& other authors (2005). Staphylococcus aureus bacteremia in patients with prosthetic devices: costs and outcomes. Am J Med 118, 1416.e19-1416.e24.

Cosgrove SE, Fowler VG. (2008) Management of methicillin-resistant Staphylococcus aureus bacteremia. Clin Infect Dis 46, S386-S393.

Costa MOC, Beltrame CO, Ferreira FA, Botelho AMN, Lima NCB, Souza RC, de Almeida LGP, Vasconcelos ATR, Nicolás MF, Figueiredo AMS. (2013). Complete genome sequence of a variant of the methicillin-resistant Staphylococcus aureus ST239 lineage, strain BMB9393, displaying superior ability to accumulate icaindependent biofilm. Genome Announc 1, e00576-13 doi: 10.1128/genomeA.0057613.

Costerton JW, Stewart PS, Greenberg EP. (1999). Bacterial biofilms: a common cause of persistent infections. Science 284, 1318-22.

Cramton SE, Gerke C, Schnell NF, Nichols WW, Götz F. (1999). The intercellular adhesion (ica) locus is present in Staphylococcus aureus and is required for biofilm formation. Infect Immun 67, 5427-33. 
Cramton SE, Gotz F. (2004). Biofilm development in Staphylococcus. In: Ghannoum, O'Toole GA, editors. Microbial biofilms. Washington, D.C. (USA): ASM Press;63-84

Croes S, Deurenberg RH, Boumans ML, Beisser PS, Neef C, Stobberingh EE. (2009). Staphylococcus aureus biofilm formation at the physiologic glucose concentration depends on the S. aureus lineage. BMC Microbiol 9, 229. doi: 10.1186/1471-2180-9-229.

Deurenberg RH, Vink C, Kalenic S, Friedrich AW, Bruggeman CA, Stobberingh EE. (2007). The molecular evolution of methicillin-resistant Staphylococcus aureus. Clin Microbiol Infect 13, 222-35.

Deurenberg RH, Rijnders MI, Sebastian S, Welling MA, Beisser PS, Stobberingh EE. (2009). The Staphylococcus aureus lineage-specific markers collagen adhesin and toxic shock syndrome toxin 1 distinguish multilocus sequence typing clonal complexes within spa clonal complexes. Diagn Microbiol Infect Dis 65, 116-22.

Donlan RM. (2002). Biofilms: Microbial life on surfaces. Emerg Infect Dis 8, 881890

Enright MC, Day NPJ, Davies CE, Peacock SJ, Spratt BG. (2000). Multilocus sequence typing for characterization of methicillin-resistant and methicillinsusceptible clones of Staphylococcus aureus. J Clin Microbiol 38, 1008-1015

Fitzpatrick F, Humphreys H, O'Gara JP. (2005). Evidence for icaADBCindependent biofilm development mechanism in methicillin-resistant Staphylococcus aureus clinical isolates. J Clin Microbiol 43, 1973-6

Harmsen D, Claus H, Witte W, Rothgänger J, Turnwald D, Vogel U. (2003). Typing of methicillin-resistant Staphylococcus aureus in a university hospital setting by using novel software for spa repeat determination and database management. $J$ Clin Microbiol 41, 5442-5448

Indrawattana N, Sungkhachat $\mathbf{O}$, Sookrung N, Chongsa-nguan M, Tungtrongchitr A, Voravuthikunchai SP, Kong-ngoen T, Kurazono H, Chaicumpa W. (2013). Staphylococcus aureus clinical isolates: antibiotic susceptibility, molecular characteristics, and ability to form biofilm. Biomed Res Int 314654 doi: $10.1155 / 2013 / 314654$.

Jansen van Rensburg MJ, Eliya Madikane V, Whitelaw A, Chachage M, Haffejee S, Gay Elisha B. (2011). The dominant methicillin-resistant Staphylococcus aureus clone from hospitals in Cape Town has an unusual genotype: ST612. Clin Microbiol Infect 17, 785-92.

Kuhn G, Francioli P, Blanc DS. (2006). Evidence for clonal evolution among highly polymorphic genes in methicillin-resistant Staphylococcus aureus. J Bacteriol 188, 169-78. 
Kwon AS, Park GC, Ryu SY, Lim DH, Lim DY, Choi CH, Park Y, Lim Y. (2008). Higher biofilm formation in multidrug-resistant clinical isolates of Staphylococcus aureus. Int J Antimicrob Agents 32, 68-72.

Lim Y, Shin HJ, Kwon AS, Reu JH, Park G, Kim J. (2013). Predictive genetic risk markers for strong biofilm-forming Staphylococcus aureus: fnbB gene and SCCmec type III. Diagn Microbiol Infect Dis 76, 539-41.

Lindsay JA, Moore CE, Day NP, Peacock SJ, Witney AA, Stabler RA, Husain SE, Butcher PD, Hinds J. (2006). Microarrays reveal that each of the ten dominant lineages of Staphylococcus aureus has a unique combination of surface-associated and regulatory genes. J Bacteriol 188, 669-76.

Lowy FD. (1998). Staphylococcus aureus infections. N Engl J Med 339, 520-532.

Mack D, Fischer W, Krokotsch A, Leopold K, Hartmann R, Egge H, Laufs R. (1996). The intercellular adhesin involved in biofilm accumulation of Staphylococcus epidermidis is a linear beta-1,6-linked glucosaminoglycan: purification and structural analysis. J Bacteriol 178, 175-83.

McDougal LK, Steward CD, Killgore GE, Chaitram JM, McAllister SK, Tenover FC. (2003). Pulsed-field gel electrophoresis typing of oxacillin-resistant Staphylococcus aureus isolates from the United States: establishing a national database. J Clin Microbiol 41, 5113-20.

Melles DC, Gorkink RF, Boelens HA, Snijders SV, Peeters JK, Moorhouse MJ, van der Spek PJ, van Leeuwen WB, Simons G, Verbrugh HA \& other authors (2004). Natural population dynamics and expansion of pathogenic clones of Staphylococcus aureus. J Clin Invest 114, 1732-40.

Milheirico C, Oliveira DC, de Lencastre H. (2007). Update to the multiplex PCR strategy for assignment of mec element types in Staphylococcus aureus. Antimicrob Agents Chemother 51, 3374-3377

Moodley A, Oosthuysen WF, Dusé AG, Marais E. (2010). Molecular characterization of clinical methicillin-resistant Staphylococcus aureus isolates in South Africa. J Clin Microbiol 48, 4608-11.

O'Neill E, Pozzi C, Houston P, Smyth D, Humphreys H, Robinson DA, O'Gara JP. (2007). Association between methicillin susceptibility and biofilm regulation in Staphylococcus aureus isolates from device-related infections. J Clin Microbiol 45, 1379-1388.

O'Neill E, Pozzi C, Houston P, Humphreys H, Robinson DA, Loughman A, Foster TJ, O'Gara JP. (2008). A novel Staphylococcus aureus biofilm phenotype mediated by the fibronectin-binding proteins, FnBPA and FnBPB. J Bacteriol 190, 3835-3850.

Oosthuysen WF, Orth H, Lombard CJ, Sinha B, Wasserman E. (2013). Population structure analyses of Staphylococcus aureus at Tygerberg Hospital, South 
Africa, reveals a diverse population, a high prevalence of Panton-Valentine leukocidin genes, and unique local methicillin-resistant S. aureus clones. Clin Microbiol Infect [Epub ahead of print] doi: 10.1111/1469-0691.12452.

Orth H, Dreyer ZS, Makgotlho E, Oosthuysen W, Sinha B, Wasserman E. (2013). Characterisation of Staphylococcus aureus bacteraemia at Tygerberg hospital. South Afr J Epidemiol Infect 28, 22-27.

Perovic O, Koornhof H, Black V, Moodley I, Duse A, Galpin J. (2006). Staphylococcus aureus bacteraemia in two academic hospitals in Johannesburg. S Afr Med J 96, 714-717.

Smith K, Perez A, Ramage G, Lappin D, Gemmell CG, Lang S. (2008). Biofilm formation by Scottish clinical isolates of Staphylococcus aureus. J Med Microbiol 57, 1018-1023. 


\section{ADDENDUM}

Declaration of contribution to laboratory work

The biofilm assay was performed by Preneshni Naicker.

Clinical data collection and folder reviews were conducted by Preneshni Naicker.

PFGE, MLST, spa typing and SCCmec typing was performed by Karayem Karayem. 
